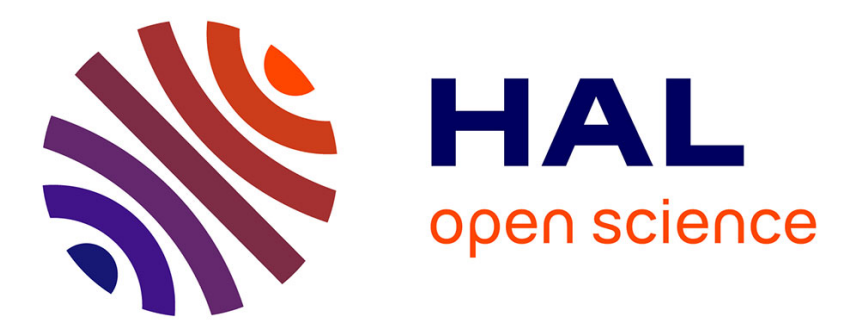

\title{
Modelling of UWB antenna perturbed by human phantom in spherical harmonics space
}

Meriem Mhedhbi, Stéphane Avrillon, Troels Pedersen, Bernard Uguen

\section{To cite this version:}

Meriem Mhedhbi, Stéphane Avrillon, Troels Pedersen, Bernard Uguen. Modelling of UWB antenna perturbed by human phantom in spherical harmonics space. EUCAP 2014, Apr 2014, The Hague, Netherlands. 10.1109/EuCAP.2014.6902600 . hal-01122228

\section{HAL Id: hal-01122228 \\ https://hal.science/hal-01122228}

Submitted on 3 Mar 2015

HAL is a multi-disciplinary open access archive for the deposit and dissemination of scientific research documents, whether they are published or not. The documents may come from teaching and research institutions in France or abroad, or from public or private research centers.
L'archive ouverte pluridisciplinaire HAL, est destinée au dépôt et à la diffusion de documents scientifiques de niveau recherche, publiés ou non, émanant des établissements d'enseignement et de recherche français ou étrangers, des laboratoires publics ou privés. 


\title{
Modelling of UWB Antenna Perturbed by Human Phantom in Spherical Harmonics Space
}

\author{
Meriem Mhedhbi ${ }^{1}$, Stéphane Avrillon ${ }^{1}$, Troels Pedersen ${ }^{2}$, Bernard Uguen ${ }^{1}$, \\ 1 IETR, University of Rennes 1, France, meriem.mhedhbi, stephane.avrillon, bernard.uguen@univ-rennes1.fr \\ 2 Department of Electronic Systems, Aalborg University, Denmark, troels@es.aau.dk
}

\begin{abstract}
The spherical harmonics expansion is an efficient tool to represent the antenna radiation pattern, especially in physical simulator, where the knowledge of full antenna data is fundamental and computationally intensive as well. Thus, the SH become imperative and represent compactly the antenna by reducing the data into a small number of coefficients. In Body Area Network (BAN) context, the goal is to go further than a compact storage of antenna data. In fact, we aim to study the effect of human phantom presence on the antenna in terms of spherical harmonics coefficients. The proposed paper will present a simple SH coefficients model allowing to predict the antenna behaviour disrupted by a human phantom, based on the knowledge of the SH coefficient of antenna in free space and antenna-phantom distance.
\end{abstract}

Index Terms-UWB antenna, spherical harmonics, Body Area Networks, measurement.

\section{INTRODUCTION}

In the context of modeling and simulation of the radio channel experienced in a wireless body area network (WBAN), the knowledge of the antenna radiation pattern is important. Moreover, a nearby body affects the antenna pattern and should therefore be included in the simulation model. However, this poses a number of challenges.

Firstly, if relying directly on measurements of the antenna alone, the obtained radiation pattern should be adapted to include the presence of the human body. For this purpose, simple models describing the relation between simple parameters such as antenna-body distance and body size would be of importance. Secondly, if relying on measurements of the combined of an antenna and body yields a new data set for each pair of antennas and bodies. The potential large number of combinations of body parts, distances, and antennas, would require a large database of antenna measurement data, which is inconvenient in such a simulator. It is therefore natural to consider models in which the antenna pattern for each antenna body configuration can be represented by only few coefficients and where the pattern reconstruction is straightforward.

In this work we approach the antenna data storage problem by means of expansion of the pattern in spherical harmonics (SH) [1], [2], [3]. In the $\mathrm{SH}$ basis, the full antenna data can be compressed to only a few coefficients. Traditionally, vector spherical harmonic (VSH) expansion have been the most popular basis for representation of far-field antenna radiation patterns because it preserves inherently the transversal field. While this feature is attractive, the VSH basis has the drawback of more complicated basis functions burdening the reconstruction task. We follow the alternative approach proposed in [4] of expanding the radiation pattern in terms of scalar spherical harmonics (SSHs).

In the present contribution, we study the effect of the human body presence close to the antenna in terms of SSH coefficients variation. In particular we focus on the change in the expansion coefficients as a function of phantom-antenna distance. We propose and validate a model for the $\mathrm{SSH}$ coefficients able to predict the observed variation of antenna radiation pattern.

\section{Measurement and Post-Processing}

\section{A. Measurement Setup}

For the study, we designed a measurement campaign specifically to study the antenna response variation in the presence of a dielectric cylinder acting as a human phantom for different antenna/phantom distances $(d)$. The antenna radiation pattern was measured in a SATIMO SG32 near-field measurement chamber at INSA Rennes. As depicted in Fig. 1, the presence of a human arm was mimicked by a phantom mounted in the vicinity of the antenna using a custom-made platform of dielectric foam with relative permittivity close to unity. The phantom consists of a nearly cylindrical plastic bottle with of radius $35 \mathrm{~mm}$ filled with MSL1800 phantom liquid. The size and shape of the phantom was chosen to represent a human arm, while still being lighted enough for the platform to support it. The bottle was fitted with a screw in the bottom to allow for mounting on the platform groove or holes. A detailed description of the measurement setup is available in [5].

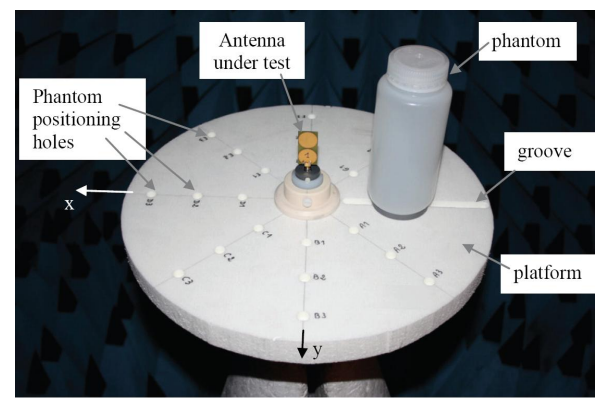

Fig. 1. Measurement Platform 


\section{B. Extraction of Scalar spherical Harmonics Coefficients}

The obtained data consists of a far-field pattern in Cartesian coordinates systems denoted by $\mathbf{F}_{\mathbf{c}}(\theta, \phi, f)$ at co-elevation $\theta$ and azimuth $\phi$, for each frequency $f$. We used a regular directional sampling grid given by:

$$
\left(\theta_{i}, \phi_{j}\right)=\left(\frac{i}{N_{t}}, \frac{2 j}{N_{p}}\right) \pi
$$

where $0 \leq i \leq N_{t}, 0 \leq j<N_{p}, N_{t}$ and $N_{p}$ are the numbers of $\theta$ and $\phi$ samples respectively.

Following [4], [6], the far-field radiation pattern represented in Cartesian systems and expanded in terms of SSHs with vector coefficients:

$$
\mathbf{F}_{\mathbf{c}}(\theta, \phi, f)=\left(\begin{array}{l}
F^{x} \\
F^{y} \\
F^{x}
\end{array}\right)=\sum_{l=0}^{L} \sum_{m=-l}^{l} \mathbf{c}_{l m}(f) Y_{l}^{m}(\theta, \phi),
$$

where the SSH function of level $l$ and mode $m$ is defined by:

$$
Y_{l}^{m}(\theta, \phi)=\sqrt{\frac{(2 l+1)(l-m) !}{4 \pi(l+m) !}} P_{l}^{m}(\cos \theta) e^{i m \phi}
$$

with $P_{l}^{m}$ denoting the associated Legendre polynomials of level $l$ and mode $m$. We limit the expansion to a maximum level denoted by $L$.

The SSH vector coefficients are obtained via least squares as follows. At each directional grid point, the antenna response is a three-dimensional complex vector. We arranging these $N_{t} N_{p}$ vectors as columns the matrix $\mathbf{F}_{\mathbf{c}}$ of dimension $3 \times\left(N_{t} N_{p}\right)$. With this definition, the expansion in (2) reads:

$$
\mathbf{F}_{\mathbf{c}}=\mathbf{C Y}
$$

where the entries of the $\left((1+L)^{2}, N_{t} \times N_{p}\right)$ matrix $\mathbf{Y}$, are the spherical harmonic functions evaluated at the sampling grid points. The matrix, $\mathbf{C}$ is of dimension a $\left(3,(1+L)^{2}\right)$ with the $\mathbf{c}_{l m}$ as columns arranged accordingly. Estimates of the coefficients $\mathbf{c}_{l m}$ are obtained in a straight forward manner by the lest square method

$$
\hat{\mathbf{C}}=\mathbf{F}_{\mathbf{c}} \mathbf{Y}^{+},
$$

where $\mathbf{Y}^{+}$is the Moore-Penrose pseudo-inverse of $\mathbf{Y}$.

\section{DATA ANALYSiS AND OBSERVATIONS FROM MEASUREMENTS}

In this section we are studying the effect of human phantom at different configurations in terms of antenna radiation pattern, Figure 2, and SH coefficients, Figure 3. Especially, the SH coefficients $\mathbf{C}(d, f)$ are investigated in terms of number of coefficients and magnitude. In the following, most important observations,represent the key of the modelling task later,are presented.

\section{A. Observation 1: Number of significant modes representing the antenna pattern}

From Figure 2 we observe that antenna pattern is radiating mostly in the half space opposite to the phantom and converges to the free space case when $d$ increases. This behaviour is expected considering the omnidirectional nature of the antenna. Besides, some ripples around the free space case are observed. Hence the pattern converge to the free space case by recovering the omnidirectional behaviour and oscillating around the free space case. These ripples might be the origin of the increase of modes number to represent the perturbed antenna shown in Figure 3.

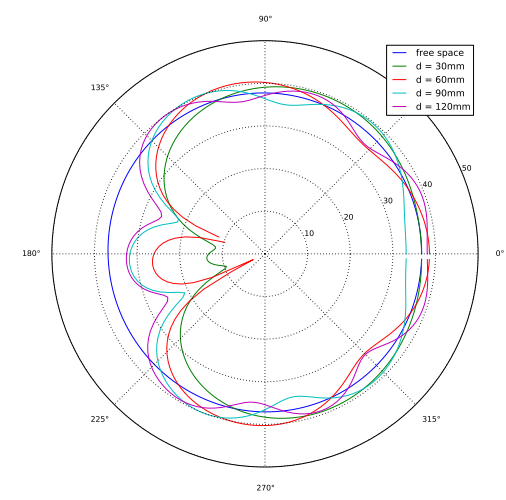

Fig. 2. Antenna radiation pattern at $3.1 \mathrm{GHz}$ for different phantom configurations $(x, y)$ plane.
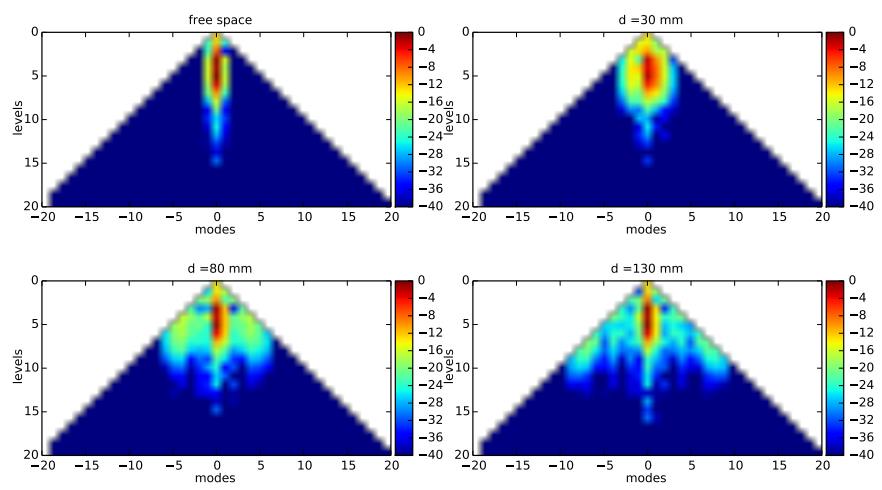

Fig. 3. Magnitude of SSH coefficients $\left(\left\|c_{l m}\right\|\right)$ at $3.1 \mathrm{GHz}$ in free space and with phantom at different distances

The increase of coefficient number might be explained differently. In fact, the number of coefficient depends on antenna dimensions [6],[7]. By introducing the phantom, an equivalent antenna (antenna+phantom) may be considered which dimensions increase with antenna/phantom distance. 


\section{B. Observation 2: Omnidirectional mode 0 energy variation}

Mode 0 energy $E_{0}$ refers to energy provided by coefficients $C_{l 0}$, where $0 \leq l \leq L$. Figure 4 shows the evolution of $E_{0}$ for the different measured $d$. This mode energy takes it is minimum value when the phantom is too close to the antenna and increases with $d$. As the phantom approaches the antenna the pattern is becomes increasingly directional, and since mode 0 coefficients represent the omnidirectional contribution which is required by increasing $d, E_{0}$ is increasing as well.

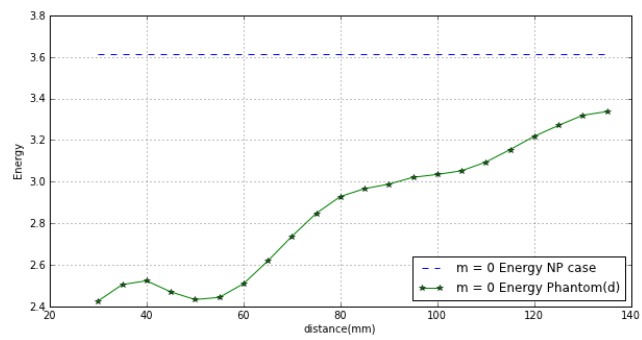

Fig. 4. Mode 0 energy w.r.t antenna/phantom distance

\section{Observation 3: Coefficient correlation functions}

As presented in previous section (Fig.3) the presence of the phantom induces the appearance of new coefficients. In order to achieve the modelling task, the origin of the new coefficients needs to be identified. Therefore the correlation functions, equation 7 , are studied in order to identify a possible relation between free space and perturbed antenna coefficients. The coefficients are indexed according to two integers $l$ and $m$ and to compute correlation functions we aggregate into one single index $k$. The equation 6 sequence the coefficients as follows (gathering all modes for different levels):

$$
\begin{gathered}
m=[0,0, \ldots, 0,1,1, \ldots, 1, \ldots, L,-L, \ldots,-1,-1, \ldots,-1] \\
l=[0,1, \ldots, L, 1,2, \ldots, L, \ldots, L, L, \ldots ., 1,2, \ldots ., L] \\
k=\frac{m(2 L+1-m)}{2}+l \\
R(n)=\sum_{k=0}^{(1+L)(2+L) / 2} C^{f s}(k) \overline{C^{d}(k+n)}
\end{gathered}
$$

where $C^{f s}(k)$ are free space antenna coefficients, $C^{d}(k+n)$ are coefficients with phantom at distance $d$ and - is the conjugate operator.

The Fig. 5 represents the auto and cross correlation functions between coefficients at maximum level $L=20$ and is computed between coefficient $\mathbf{C}_{\mathbf{l m}}$, where $m \geq 0$ because of the relation $c_{l-m}=(-1)^{m} \overline{c_{l m}}$.

The Fig. 5 exhibit a periodic behaviour in cross correlation functions $(\mathrm{CCF})$. The cross correlation is computed between free space and perturbed antenna coefficients. Since the free space antenna is represented by modes $m=[0,1]$ and the
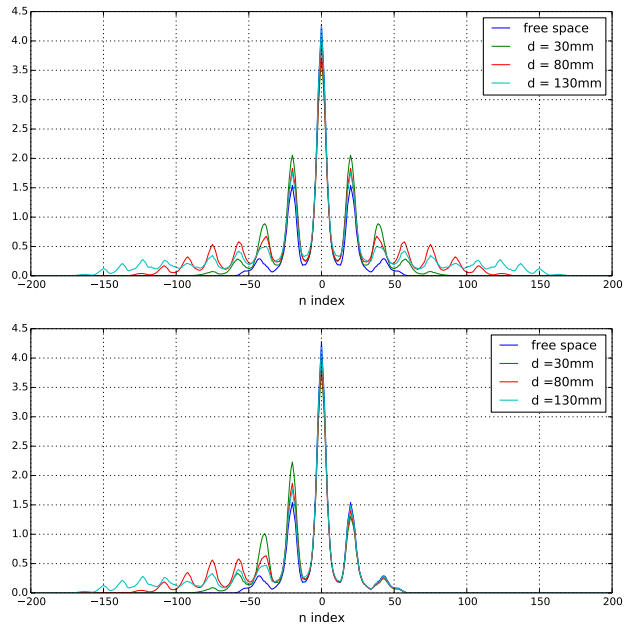

Fig. 5. Auto correlation coefficient function (top), cross correlation function between coefficient in free space and in phantom presence (bottom)

number of peaks in CCF is higher than 2 and even more increases with the distance(same behaviour of modes number), we assume that there is a strong relation between the new coefficients and free space coefficients.

\section{Proposed Model Structure}

A SH coefficient model able to anticipate the pattern of a disrupted antenna by a human phantom is proposed in this part. The main goal of this model is to make the channel modelling task in physical simulators easier. Based on both knowledge of antenna SH coefficients in free space and the antenna-phantom distance, the challenge is to generate the $\mathrm{SH}$ coefficients of the perturbed antenna (Figure 6).

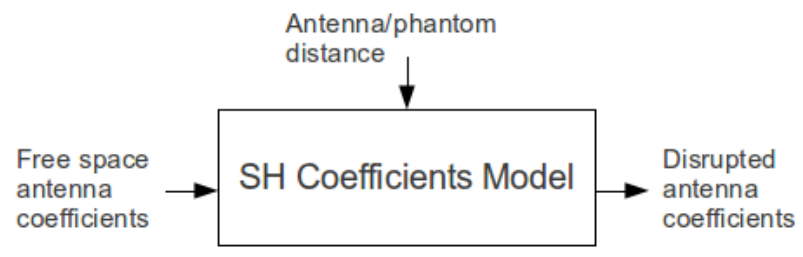

Fig. 6. Model Scheme

As the observations show the presence of the phantom around the antenna induces the appearance of new modes (coefficients). Thus, in the model elaboration task we start by computing the number of modes necessary to represent antenna pattern, then studying the free space coefficients and finally the new coefficients are studied. 
a) Number of modes: The presence of the phantom induces the appearance of the new coefficients (modes), therefore the modelling task treat separately both types of coefficient (free space and new coefficients). However, before coefficients modelling, the number of modes $M$ should be identified. Based on observation 1, $M$ varies proportionally with the distance, and using a stair case fitting $M$ is determined, Figure7.

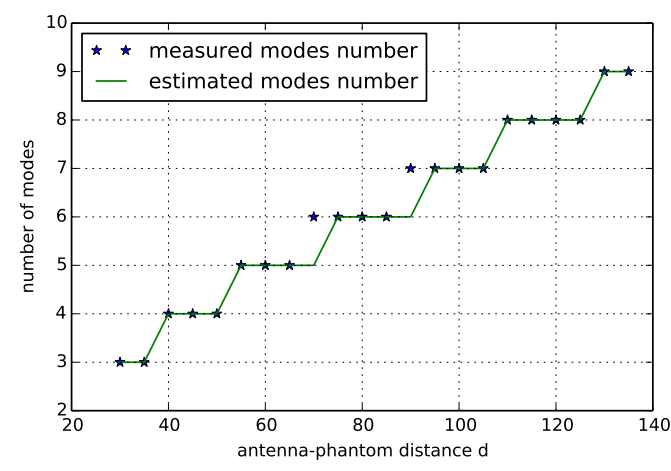

Fig. 7. Number of modes w.r.t antenna/phantom distance $d$

Another parameter should be introduced $M_{0}$ which is the maximum mode representing the free space antenna. We notice that for a pure omnidirectional pattern $M_{0}=0$, but for measured antennas higher modes $M_{0}=[1,2]$ can be observed. It depends on antenna pattern and measurement conditions.

b) $\mathbf{m} \leq \mathbf{M}_{\mathbf{0}}$ : The Spherical harmonics coefficient $C_{l m}$ where $m \leq M_{0}$ represents the free space antenna coefficients whose behaviour is generally omnidirectional.The perturbation of these coefficients decrease with the antenna/phantom distance $d$. Based on observation 2 representing the variation of mode 0 energy (which is generally the most significant modes) we assume that the perturbation caused by the phantom is multiplicative and varies linearly with the distance.

c) $\mathbf{m}>\mathbf{M}_{\mathbf{0}}$ : The Spherical harmonics coefficient $C_{l m}$ where $m>M_{0}$ represents the coefficients that appear due to the phantom presence. This subtask of coefficient modelling is an extrapolation since these coefficient do not exist in free space. To be able to predict their behaviour we studied the cross correlation functions. Based on observation 3 which reveals a strong correlation between free space modes and new modes we propose that these coefficients represent a mitigated and shifted copy from the highest mode $\left(M_{0}\right)$ representing free space antenna.

Based on observations and data analysis presented in previous, the proposed model structure is the following:

$$
C_{l m}(f, d)=\left\{\begin{array}{c}
A_{m}(d) C_{l m}^{f s}(f), m \leq M_{0} \\
A_{m}(d) C_{l M_{0}}^{f s}(f), m>M_{0}
\end{array}\right.
$$

where $d$ is antenna/phantom distance, $f$ is the frequency, $C_{l m}(f, d)$ are disrupted antenna SH coefficients, $C_{l m}^{f s}(f)$ are free space antenna coefficients, and $A_{m}(d)$ represents the
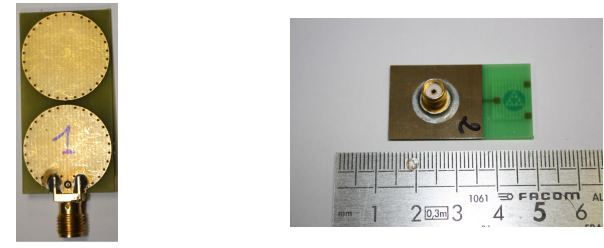

Fig. 8. Thomson 1 (left), fractal structure (right) antennas

\begin{tabular}{|l|l|l|}
\hline Parameters & Values $\left(m \leq M_{0}\right)$ & Values $\left(m>M_{0}\right)$ \\
\hline$a_{m}$ & 0.002 & -0.002 \\
\hline$b_{m}$ & 0.55 & 1.55 \\
\hline$c_{m}$ & 0 & -0.006 \\
\hline$d_{m}$ & 0 & 1.22 \\
\hline
\end{tabular}

TABLE I

MOdel PARAMETERS NUMERICAL VALUES

perturbation:

$$
A_{m}(d)=\alpha_{m}(d) \exp \left(j \phi_{m}(d)\right)
$$

where $\alpha_{m}(d)$ represents the real perturbation which varies linearly with the distance $d$ (observation 2) and $\phi_{m}(d)$ is a shift introduced introduced on disturbed coefficient.

The real perturbation $\alpha_{m}(d)$ varies linearly with the distances, this choice is based on observation where the linear approximation seems to fit well the variation. But the structure depends on the modes:

$$
\alpha_{m}(d)=\left\{\begin{array}{l}
a_{m} d+b_{m}, m \leq M_{0} \\
\frac{\left(a_{m} d+b_{m}\right)}{m-M_{0}}, m>M_{0}
\end{array}\right.
$$

The free space coefficients $\left(m \leq M_{0}\right)$ depend only on the distance $d$ which means the recovery of the free space structure and behaviour. The new coeffiecients $\left(m>M_{0}\right)$ depend on the mode $m$ and the distance $d$. In fact, the number of cofficient increases with the distance but not their values or effect which clear in Figure 3 (coefficients values) and Figure 5 (correlation functions).

d) $\phi_{m}(d)$ : Regarding the shift $\phi_{m}(d)$, it represents the shift between the different modes and follows a linear variation and depends on $d$ and the mode $m$ :

$$
\begin{gathered}
\phi_{m}(d)=m\left(c_{m} d+d_{m}\right) \\
\text { V. RESULTS }
\end{gathered}
$$

\section{A. Model fitting}

In this subsection the modelling results are presented for measured antenna Thomson 1 [8], in Figure 8, used to elaborate the model.

These results are also obtained using numerical values in Table 1:

As the numerical values shows, $\alpha_{m}\left(m \leq M_{0}\right)$ increases with the distance which proves the fact that the antenna recover the behaviour in free space while $\alpha_{m}\left(m<M_{0}\right)$ is decreasing with $d$ because the energy is splitted over a higher number of coefficients (modes) but their impact is reduced. 

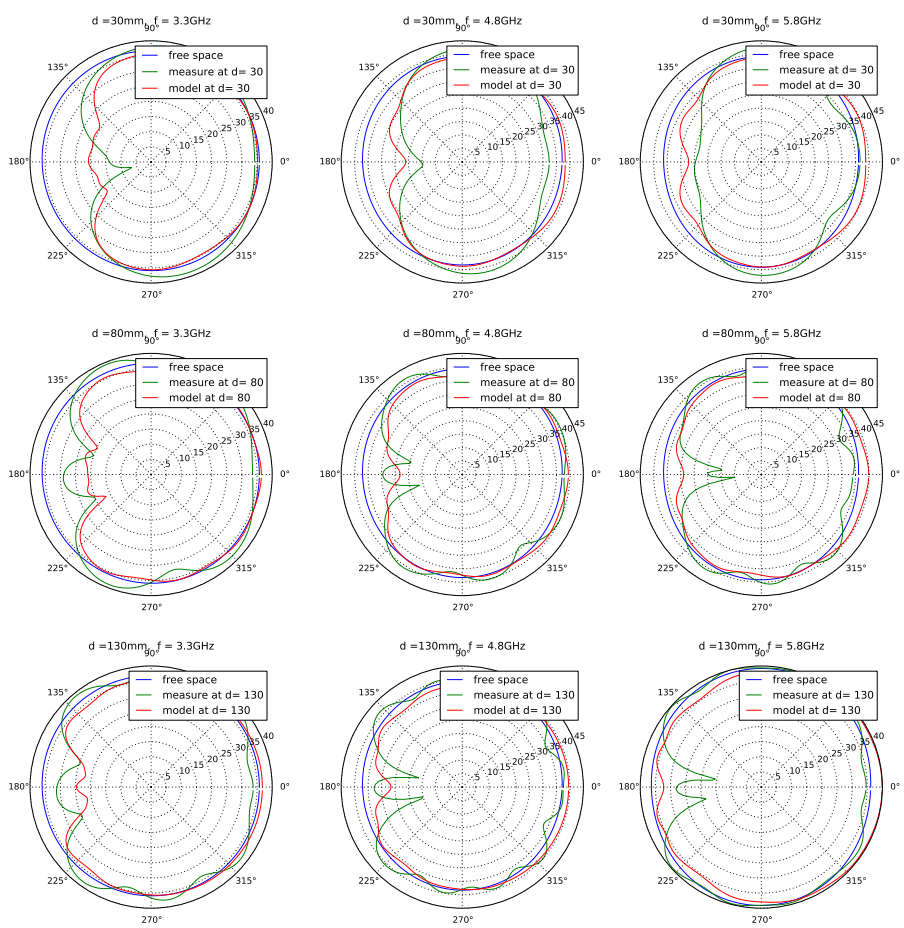

Fig. 9. Antenna pattern comparison (Thomson 1 antenna )

The value of the shift $\phi_{m}$ is decreasing with respect to the distance because it has in impact on the antenna radiation direction. Besides observations show that the value of $\phi_{m}$ depends on $m$ and at a fixed distance, it increase with modes.

The Figure 11 shows the modelling results in terms antenna pattern in the plane $\theta=\frac{\pi}{2}$ at different frequencies and antenna/phantom distances.

Thus, as the results show, the model is able to generate the global behaviour of perturbed antenna by human phantom. In fact, the half space radiation is controlled and maximum and minimum radiation directions as well and in terms of relative error $\epsilon$ (equation 12) averaged over the frequency bandwidth the results remain sufficient (around 10\%, Figure 10).

$$
\epsilon=\frac{\left\|E_{m}-E\right\|_{2}}{\|E\|_{2}}
$$

where $E_{m}$ is the field obtained from modeled coefficient and $E$ is the measured field.

The proposed model is frequency independent (we assume that the frequency dependency is in the coefficients themselves), level independent (because of the choice of measurement set: the phantom is parallel to $\mathrm{z}$ axis so the symmetry over $\phi$ is affected which means the modes) and linear (simple)is able to generate, with a sufficient reconstruction results, the set of disturbed antenna SH coefficient based on the knowledge of the free space coefficient and the antenna/phantom distance.

\section{B. Model Validation}

The same measurement campaign was carried out with another fractal structure antenna, Figure 8 , in order to validate

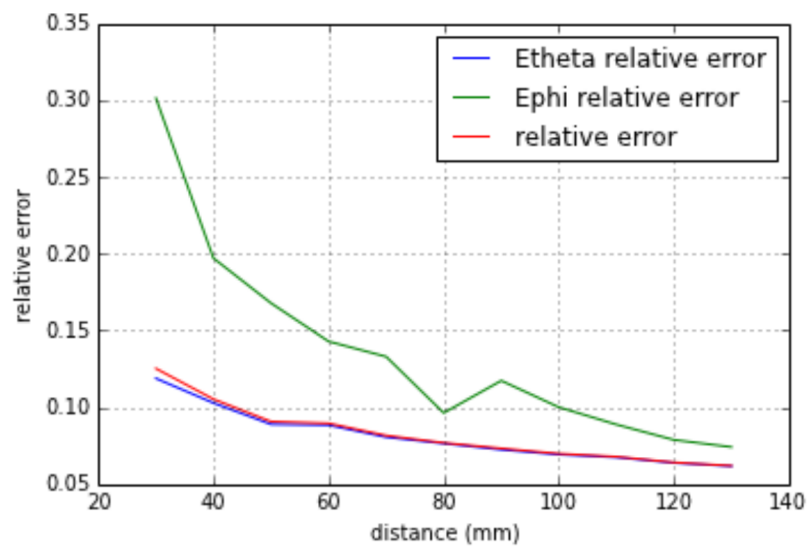

Fig. 10. Relative error (Thomson 1 antenna)
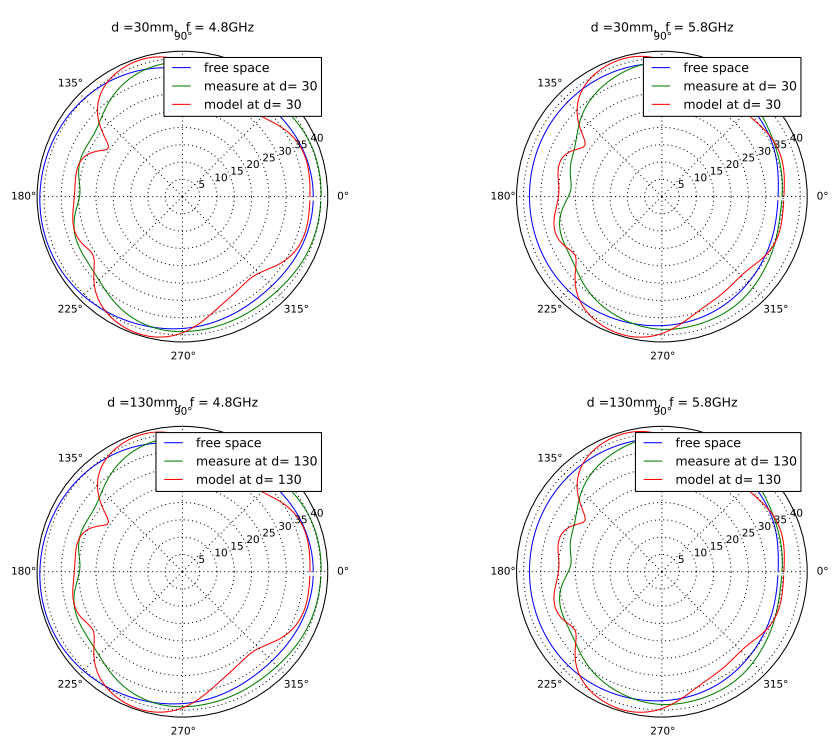

Fig. 11. Antenna pattern comparison (fractal structure antenna )

the model. In using the same model parameters the results seems to be very sufficient.

\section{CONCLUSION}

The paper presents a simple model based on spherical harmonics coefficients to represent an antenna pattern, allowing to predict a disrupted antenna behaviour by a human phantom with respect to the antenna-phantom distance. This model is going to be integrated in a channel simulator where the body is approximated by dielectric cylinders animated thanks to motion capture files. The purpose being to have fast access to a representative perturbed antenna pattern with full polarization and ultra wide bandwidth. The dependency of the model parameters with constitutive parameters of the dielectric cylinder and cylinder dimension have still to be investigated. This work has demonstrated the following practical interesting fact that simple modification of the non perturbed antenna 
coefficient can reproduce reasonably accurate reconstruction of the antenna in the presence of a dielectric cylinder. In the future work, this model will be generalized and the different parameters should take into account the volume and the dimensions of the phantom.

\section{ACKNOWLEDGMENT}

This work has been carried out in the frame of the CORMORAN project, which is funded by the French National Research Agency (ANR) under the contract number ANR-11INFR-010 and the framework of the ICT project ICT-248894

WHERE2, which is partly funded by the European Union.

\section{REFERENCES}

[1] C. Roblin. Ultra compressed parametric modelling of uwb antenna measurements. In Antennas and Propagation, 2006. EuCAP 2006. First European Conference on, pages 1-8, 2006.

[2] R. Burghelea, S. Avrillon, and B. Uguen. Vector spherical harmonics antenna description for ir-uwb ray tracing simulator. In Electromagnetics in Advanced Applications, 2009. ICEAA '09. International Conference on, pages 303-306, 2009.

[3] R. Burghelea, S. Avrillon, and B. Uguen. Ultra-compact parametric modelling of three-dimensional antenna radiation pattern for impulse radio-ultra-wide band ray tracing. Microwaves, Antennas Propagation, IET, 6(11):1251-1258, 2012.

[4] J. Rahola, F. Belloni, and A. Richter. Modelling of radiation patterns using scalar spherical harmonics with vector coefficients. In Antennas and Propagation, 2009. EuCAP 2009. 3rd European Conference on, pages 3361-3365, 2009.

[5] Troels Pedersen Marios Raspopoulos Ronald Raulefs Julien Stéphan Gerhard Steinboeck Bernard Uguen Wei Wang Yoann Corre, Mohamed Laaraiedh. Fp7 where2 deliverable d1.7 http://www.ictwhere2.eu/documents/deliverables/deliverable-d1.7.pdf, July 2012.

[6] Yinchao Chen and T. Simpson. Radiation pattern analysis of arbitrary wire antennas using spherical mode expansions with vector coefficients. Antennas and Propagation, IEEE Transactions on, 39(12):1716-1721, 1991.

[7] J.E. Hansen and Institution of Electrical Engineers. Spherical Near-field Antenna Measurements. IEE electromagnetic waves series. P. Peregrinus, 1988.

[8] E. Gueguen, F. Thudor, and P. Chambelin. A low cost uwb printed dipole antenna with high performance. In Ultra-Wideband, 2005. ICU 2005. 2005 IEEE International Conference on, pages 89-92, 2005. 